\title{
Investigating The Dynamic Behavior Of Introducing Outcomes Assessments In Information Systems Programs For Accreditation Compliance
}

Hussein Fakhry, Ph.D., University of Dubai, UAE

\begin{abstract}
The success of information systems programs accreditation process is dependent on a crucial accreditation compliance requirement which is assessing programs' outcomes and using these assessments to close the loop and introduce key adjustments in the programs to raise outcomes level above an acceptable benchmark level. Furthermore, institutions would be required to maintain these assessments processes throughout the accreditation period and produce interim reports about their implementations. However, outcomes assessments feedback implementation is a complex and dynamic process which can produce unexpected and surprising results. This study investigates the different forms of assessment process and studies its dynamics by developing an academic program model with outcomes assessments feedback. These developments are based on genuine experiences with information systems program assessments and successful accreditation by ABET. The development uses system dynamics approach which can model complex and dynamic socio-technical systems. A system dynamics modeling and simulation are developed which can help academic administrators study the consequences of any assessment policy decisions in an interactive and dynamic way. Simulation runs revealed inherent oscillation of program outcomes over the years. Both the model and the discovered program outcomes oscillation are validated by analyzing actual assessments data collected over five year period. Extenuating this oscillation demands a careful design of the assessments feedback policies as well as course delivery to balance its effects. The simulation results lead to recommending guidelines to achieve effective assessments and raise the program quality. This is a novel approach to study outcomes assessments processes using the rich system dynamics approach.
\end{abstract}

Keywords: Accreditation Compliance; Information Systems Program Assessments; Outcomes Assessment Feedback; Academic Program Model; System Dynamics Modeling and Simulation

\section{INTRODUCTION}

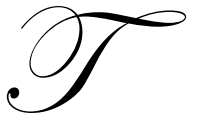

he majority of academic institutions offering information systems programs, worldwide, are striving to raise the quality of their information systems programs to achieve local and international recognition. To accomplish these goals, academic institutions seek accreditation from different accreditation bodies such as ABET (the Accreditation Board for Engineering and Technology), AACSB (the Association to Advance Collegiate Schools of Business) and others. Any accreditation body would require that its quality standards to be met by the academic institutions (ABET, 2011) and (AACSB, 2011). These standards imply that the design of academic programs and associated curricula are following what is known as Outcomes Based Education (OBE) (Allan, 1996). Such model requires extensive assessment of all learning outcomes and degree programs' objectives. These assessments identify strengths and deficiencies, and subsequently outline recommendations for corrective actions. The later would be used by the institutions to close the loop; hence introducing changes in the academic degree programs and curricula. This raises the academic standards and all outcomes and objectives above the required benchmark levels (Angelo, 1995). However, implementation of 
outcomes assessments feedback is challenging, and complex task, as there are many forms for designing and implementing the assessments process and its feedback. Furthermore, introducing assessments process would further complicate the academic program process and create unpredicted and surprising results. There is a need to study the outcomes assessment feedback process form a holistic implementation system view and from a system dynamics view. The experiences gained through the curricula design, documentation, assessment process, self-study evaluations' reports and the successful accreditation process of the Computing and Information Systems Program at University of Dubai by the Computing Accreditation Commission (CAC - ABET) in 2008 and the accreditation of the College of Business Administration by AACSB in 2009, provide the researcher valuable insights into the mechanisms of the process of outcomes assessment feedback and the anticipated performance (University of Dubai, 2011).

\section{LITERATURE REVIEW}

In the research literature on higher education, assessment is defined as "the systematic collection of data and information across courses, programs and the institution, an integral part of teaching and learning used to help both, and an essential component of a college's mission" (Morante, 2003). It is also defined as "Assessment is an ongoing process aimed at understanding and improving student learning. It involves making our expectations explicit and public; setting appropriate criteria and high standards for learning quality; systematically gathering, analyzing and interpreting evidence to determine how well performance matches those expectations and standards; and using the resulting information to document, explain and improve performance. When it is embedded effectively within larger institutional systems, assessment can help us to focus our collective attention, examine our assumptions, and create a shared academic culture dedicated to assuring and improving the quality of higher education" (Angelo, 1995).

Numerous researches on assessment and accreditation experiences confirm the value of integrating feedback actions based on Academic Program Outcomes assessment with clear merits for quality enhancement. For example refer to the researches of (Allan, 1996), (Angelo, 1995), (Morante, 2003), (Allen, 2004), (Walvoord, 2004), (Gloria, 2006), (March, 2007), (UCF, 2008), (CDU, 2009), and (AALHE, 2011).

However, most of the related literature focuses on assessment as measurements and the design of different assessment tools. It assumes that the consequent actions taken and their feedback to the academic program will yield all the required adjustments and raise the learning outcomes levels. Even when assessment is discussed or mentioned as a process as detailed in (Morante, 2003), the discussions focus on other issues such as outcomes development or curriculum alignment and not on how the feedback actions will be implemented and how they impact the learning outcomes in the long run over many years of delivering the same curricula. Therefore, there is a need to understand and analyze the impact of feedback actions, both in degree and dynamic behavior. The resultant insights could be used to advise Academic Administrators on proper assessment policies and help devise better best practices for course instructions and delivery. This is very crucial as accreditation bodies such as ABET and AACSB require institutions to continue implementing assessments processes, collect data, and report results in interim reports for the full accreditation period.

To date, no single study has focused on modeling and studying the dynamic behavior of academic programs utilizing the outcomes assessment feedback. Also, no study investigates the possible feedback structures to use in utilizing the results of the assessment process. In addition, modeling academic programs dynamic behavior has not been clearly understood and described in the literature. On the other hand, research projects on the dynamics of the learning process focused on modeling the dynamics of learning and teaching of a student in one course and in one semester (Eftekhar, Strong, \& Hawaleshka, 1997) (Eftekhar \& Strong, 1998) (Eftekhar \& Strong, 1999) (Eftekhar \& Strong, 2007). There is no consideration given to the collective behavior of courses grouped as an academic program. However, these researches of Eftekhar et al shed important light and provide insight into the dynamics of the learning process and its different factors. 


\section{THE METHOD}

This study uses the system dynamics method pioneered by Forrester which models complex social systems as networks of feedback loops with different time delays and nonlinearities (Forrester, 1958) (Forrester, 1990). It assumes two fundamental types of feedback loops; positive feedback (i.e. reinforcing) loops, mainly responsible for any social growth and negative feedback (i.e. balancing or goal seeking) loops which can balance growth and attempt to achieve a desired level of performance. All types of time delays and nonlinearities can exist in social systems. System dynamics assume two fundamental types of variables; stocks which represent the levels achieved at specific accumulations (i.e. integrations) in the system and can be regarded as the system states, and flows which are the variables responsible for introducing dynamics changes to all stocks in the system. The system structure can be represented as a Stock \& Flow diagram. Successful use of system dynamics, generally, goes through two main phases; first a qualitative research phase to develop the structure of the model, and second a quantitative research phase to choose the model parameters, develop Stock \& Flow equations, and simulates it in system dynamics software. The simulation can help develop insights into the dynamic behavior, parameters sensitivity and impact, and possible predictions of the future trends (Lane, 2007). This study follows the same approach. The following sections will introduce qualitative discussions, deductions and reference to existing research in order to build the structure of the model. The quantitative work will follow with the simulation of the model in VENSIM PLE (VENSIM, 2011). The justification for using VENSIM PLE is that it is free for academic, public research, and personal use. This study will consider different simulation run scenarios to examine the different possibilities of the learning process and assessment feedback actions e.g., weak learning process but strong assessment feedback actions and so on.

\section{Modeling Academic Programs}

The objective of this section is to develop a dynamic model of the academic program to enable investigating the long term dynamic effects of different assessment feedback polices. The first phase of developing the model begins by a high level, holistic view for the academic program teaching and learning process that focuses mainly on input - output behavior.

According to (Fincher, 1994) "Learning is a process of acquiring and integrating through a systemized process of instruction of organized experience varying forms of knowledge, skills, and understanding that the learner may use or apply in later situations and under conditions different from those of instructions". This definition illustrates the system view of learning with many input factors. It emphasizes an inherent integration process which works in a systematic way to produce learning. Furthermore, the teaching and learning process can be modeled as a black box with inputs (e.g., learning factors) and outputs (e.g., learning outcomes) (Eftekhar \& Strong, 2007). Assessment introduces a negative feedback loop around the academic program. This loop is basically a goal seeking loop which attempts to bring the academic program learning outcomes to a desired benchmark level specified by the academic institution and acceptable by the accreditation bodies e.g., $60 \%$ level. Hence, following Eftekhar (Eftekhar \& Strong, 2007), and focusing on the aggregate knowledge, skills, and behaviors of students, faculty, and the institution, the academic program can be viewed as a system with multiple inputs and multiple outputs with an outcomes assessment feedback loop as illustrated in Figure 1. Many input factors interact in the learning process, such as motivation and interest, prior knowledge of subject, background knowledge, abilities and attitudes, effort, determination, apparatus, institution's features, learning style, nature and quality of instruction, course structure and content, study skill, comfort, demand, quality of effort, productivity and environment (Eftekhar, Strong, \& Hawaleshka, 1997) (Eftekhar \& Strong, 1998) (Eftekhar \& Strong, 1999) (Eftekhar \& Strong, 2007). 


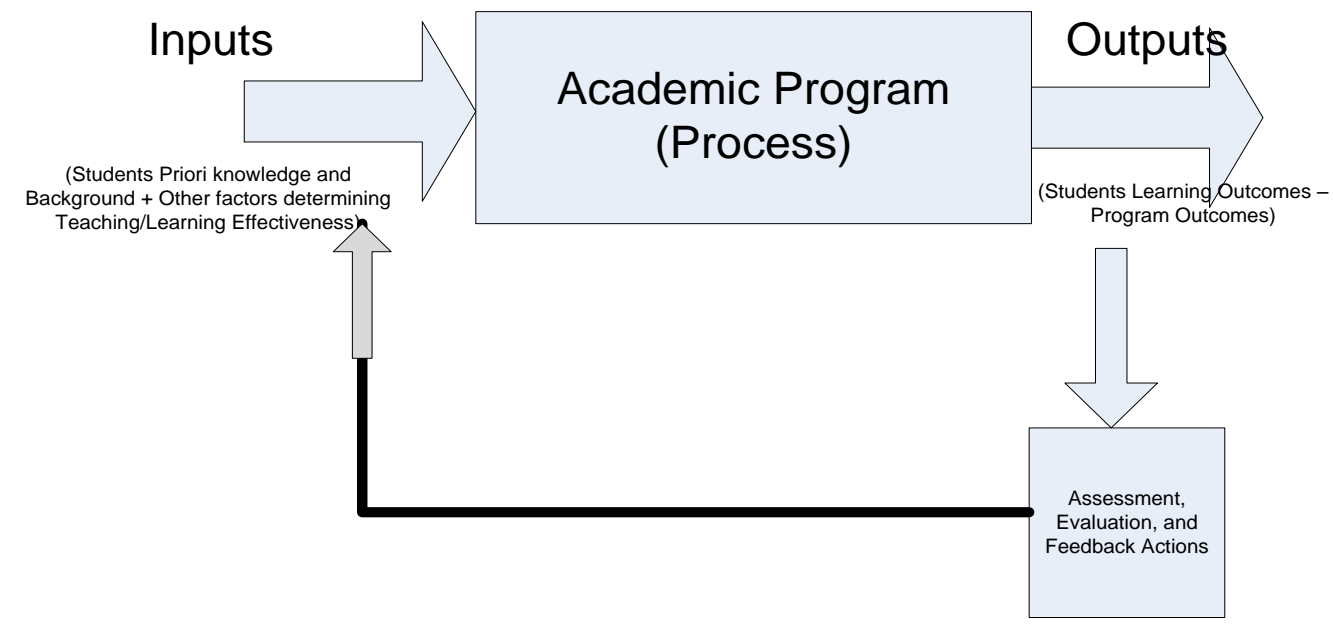

Figure 1: Fundament Model of an Academic Program with Outcomes Assessment Feedback

Figure 1 can be further divided into different units, levels or years. It is common practice to design bachelor programs as four levels (e.g., 100, 200, 300 and 400 level courses) and develop an academic model plan which distributes these courses over a four year period. This practice allows viewing the academic program (at bachelor level) as a cascade of four units (systems) as shown Figure 2. Although only the bachelor level is illustrated and discussed in this research, the developed models can be customized to the associate level or postgraduate levels.

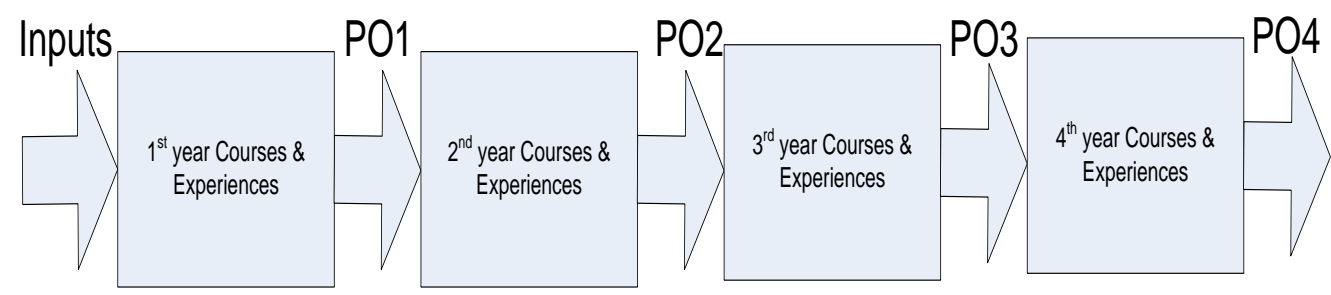

Figure 2: Academic Program as Cascade of Academic Levels / Years

The outputs for any academic level are the learning outcomes (program outcomes) $P O_{i}$ which are specific to this particular level and align with the final program outcomes $\mathrm{PO}_{4}$. These level outcomes also serve as inputs to the following higher academic level as they will represent students' prior knowledge and background when entering the higher level. The model illustrate that the final program outcomes are $\mathrm{PO}_{4}$ achieved through the achievement and accumulation of different levels' outcomes over the years.

\section{Assessment Feedback Structures}

A careful investigation of the model illustrated in Figure 2 reveals that two general feedback structures (or a hybrid modification of them) can be applied. The first general structure uses the final program outcomes assessment for feedback throughout the whole program as shown in Figure 3, which is a centralized assessment feedback with actions channeled to different courses at all levels. These actions can have a stronger weight for the fourth level courses to provide faster (short term improvements); while the feedback actions to the other levels might have a lesser weight as they are responsible for the long term improvements in the academic program. 


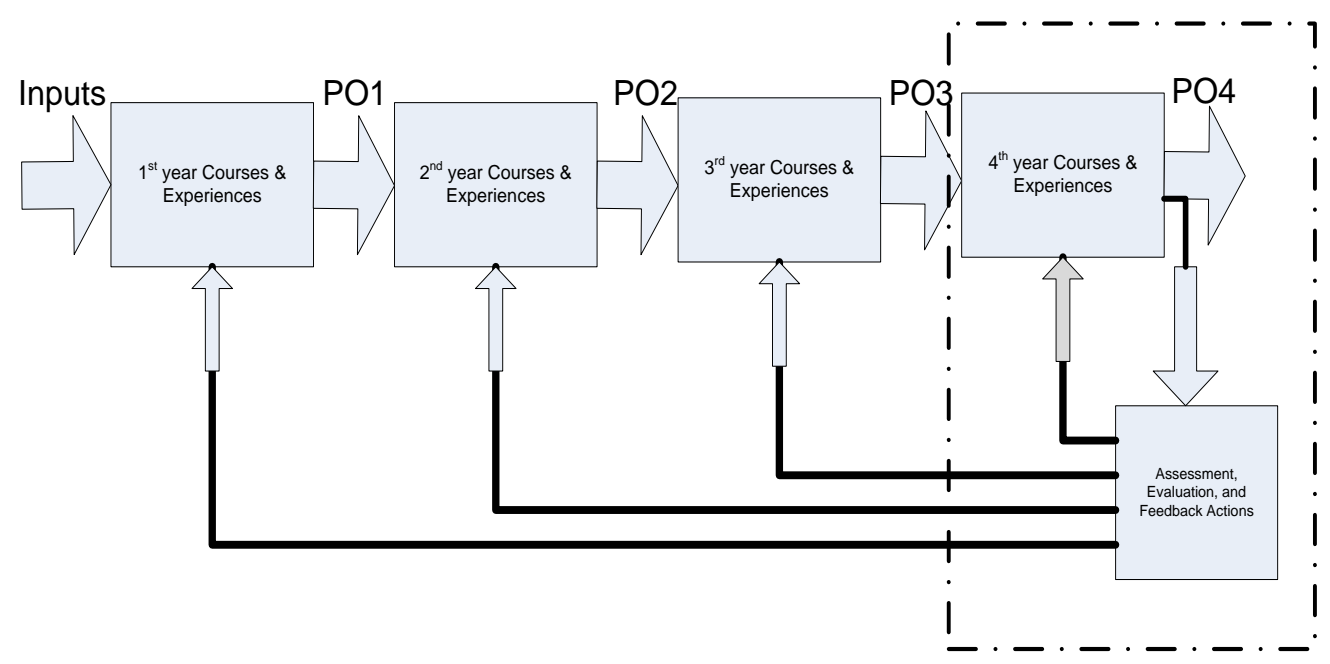

Figure 3: Centralized Outcome Assessment with feedback to all levels

The second general structure is illustrated in Figure 4. This structure focuses on outcomes assessment and feedback to each academic level, independent of the other levels. The implicit assumptions here are that all levels have their own outcomes which are perfectly aligned to support the final program outcomes. This kind of assessment is a form of distributed outcomes assessment with local feedback to its corresponding academic level. An extreme form of this structure (which is very expensive to implement), is to have outcomes assessment localized feedback in all curriculum courses.

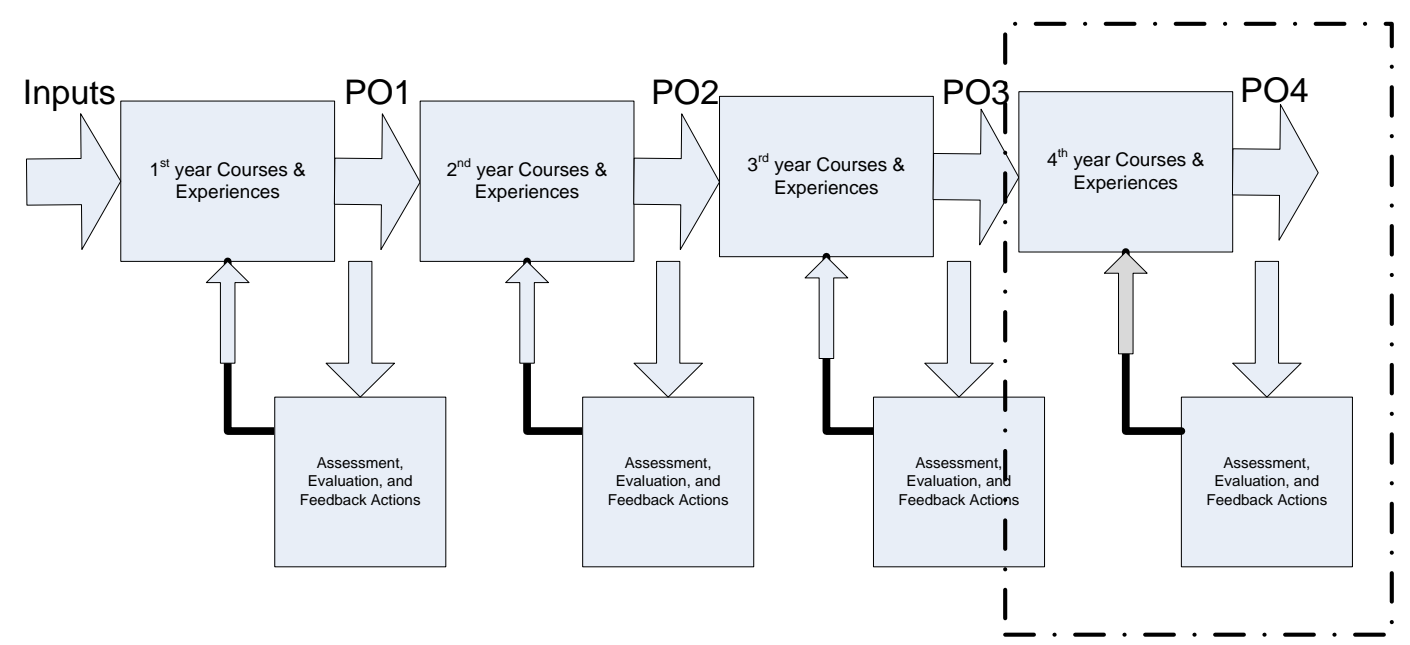

Figure 4: Distributed Outcomes Assessment with local feedback to the corresponding academic level

Regardless of which assessment feedback structure academic institutions follow, the full academic program dynamic model with outcomes assessment feedback is truly a very complex model with very large number of variables. The development of such a model is very challenging and demanding in terms of resources. However, a careful investigation of the above structures reveals that there is a key part which can be used to build the full structure. This key part is highlighted with the dashed boxes in Figure 3 and Figure 4. This part can represent one academic level, selected group of courses, module or even a course, with outcomes assessment feedback closed on it. A careful investigation and modeling of this part can represent the corner stone in building the full academic 
program dynamic model. Another interesting and valid view is that the whole academic program can be modeled as one unit resembling the mentioned corner stone part (refer to Figure 1). Therefore, it is very crucial to understand and model this part, which is the focus of the following sub-sections.

\section{Dynamic model of one academic level with assessment feedback}

The following subsections, details the development of the dynamic model for one level with outcomes assessment feedback. It discusses first the model of the academic level, then modeling the assessment feedback path and later presents the complete model using a Stock \& Flow diagram. A single box (process) is assumed to represent the collective aggregate behavior of the all courses in this academic level year. Furthermore, the simplifying assumptions regarding the inputs and the outputs are discussed.

\section{The Academic Program Dynamic Model}

The input factors to the academic program shown in Figure 1 are:

1. Students prior knowledge and background

2. Nature of the learning materials, tasks, equipment and facilities

3. Nature and quality of instructions, practice, guidance, feedback, students' assessments, etc.

4. Situational or Environmental conditions, e.g. class size, forms of reinforcement, interactions, etc. (Fincher, 1994).

For the sake of simplifying the model development, the inputs are grouped as two main inputs and are shown in Figure 5. The first input is the factor "students' prior knowledge and background" which cannot be changed and controlled through the assessment feedback for this particular level. The students' prior knowledge depends greatly on students' past knowledge, life, work experiences and their learning in previous levels. The second input represents the collective effect of the remaining three factors above which can be affected by the assessment feedback. For example, the instructional materials and use of information technology in the classroom can be altered based on feedback actions. This second input represents what can be called teaching and learning effectiveness. Thus the study focus is to model and study the dynamic relation between the Input that is the students' prior knowledge, and teaching and learning effectiveness, and the Output that is the academic program learning outcomes representing the graduates knowledge and skills at the time of completing the academic program or a particular level. Therefore the inputs are grouped as controlled input and uncontrolled input. The controlled input is named Teaching-Learning Effectiveness and covers factors 2, 3, and 4 above. The uncontrolled input is the students' prior knowledge and background. The students' prior knowledge will serve as initial condition in the simulated model later. The academic program process (i.e. the teaching and learning process) is mainly an accumulation and integration of learning experiences as the definition above suggests (Fincher, 1994). Hence, the academic program system can be viewed as shown in Figure 5.

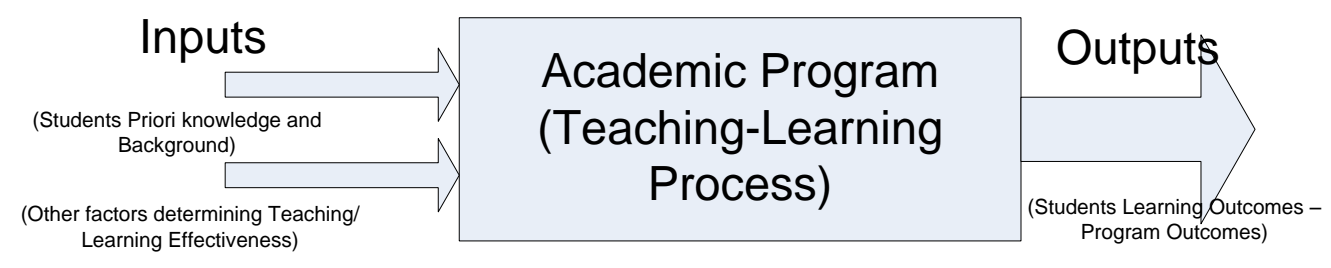

Figure 5: Academic Program with inputs divided into controlled and uncontrolled

It is usual to assume that the final program outcomes are built incrementally through the different academic levels. It can be assumed that each level contributes equally to the achievement of the final outcomes. Therefore, if the full scale for the final program outcomes level is assumed to be one hundred points, then each level scale can be twenty five points. This scale of outcomes level can be in points or percentage. The systematic progress is assumed to build students' knowledge and skills as they progress in the program. In this model, the achieved program 
outcomes at the end of the fourth level are the same as the program outcomes considered earlier. The above modeling assumptions are for a graduating class model with the academic program structured as four years. However, with a credit hour system, an academic plan over four years can use groups of courses and develop the same model view.

Assuming an ideal learning environment, the students' learning outcomes (program outcomes) will be built in an incremental manner starting from the prior knowledge entry (initial condition) value. It will reach one hundred points level for the program outcomes at the end of the fourth level or year, in ideal situations.

Figure 6 represents this understanding and assumption. Based on this ideal situation, it would be reasonable to assume that each academic year (four years in this model) will contribute a twenty five points increment assuming the worst levels initial values of outcomes at the start of the program which is $\mathrm{PO}_{0}=0$. Negative $\mathrm{PO}_{0}$ levels are not considered as typical university administration policies dictate minimum qualifications for admitted students. Based on this model every academic year can have its own maximum level as twenty five points (ideal situation), which collectively build the PO for the whole academic program. As shown in Figure 6 below, this is an implicit linear assumption which is needed to simplify the development of the dynamic model later. However, in many real situations there is a nonlinear contribution as later courses in the curriculum tend to have higher contribution as they integrate knowledge and skills gained through the curriculum. This nonlinear contribution can be specifically examined in a future modification of the model.

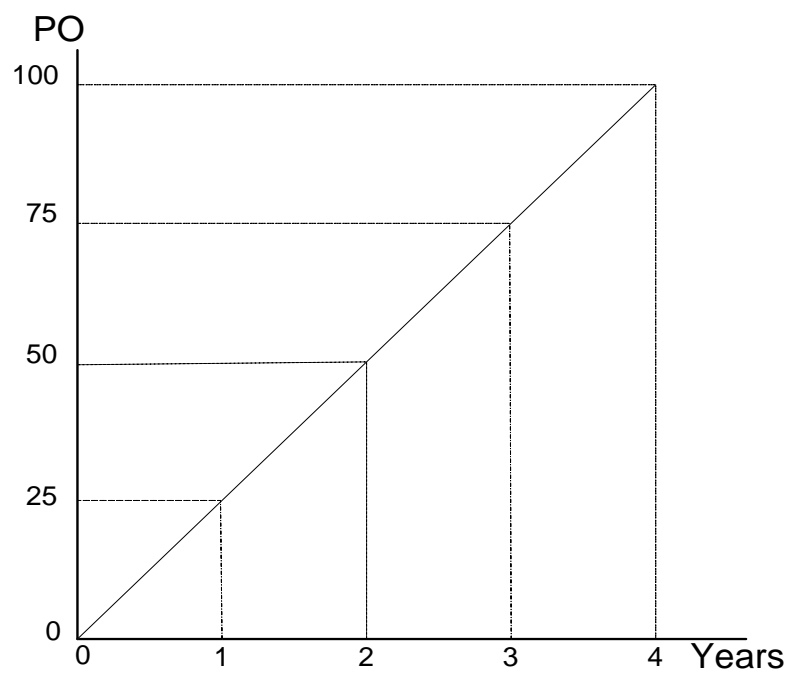

Figure 6: The ideal progress of Program Outcomes development

To further simplify the development of this first model, the different learning outcomes are grouped and represented by one learning outcomes index, which is a weighted average of the group of program outcomes. This index is named $P O_{i}$ where $i$ represent the academic year. The collective and aggregate dynamic behavior of each academic level is to build and accumulate program outcomes index level over one academic year.

If learning experiences are accumulated at a reasonable weight and rate, the program learning outcomes index will grow steadily to reach the target by the end of the year. Mathematically, this is the behavior of stock, accumulator, or integrator with a constant inflow or rate to integrate.

Its mathematical model as integration is; 
$P O_{i}=\int_{0}^{t=1} w_{i} d t$

Where, the symbol $i$ represents any academic level (e.g., 1, 2, 3, or 4); $P O_{i}$ represents the learning outcomes index for level $i$, while $w_{i}$ represents the learning-teaching rate. With this model, it can be assumed that the full academic program is a cascade of integrators with different weights for different academic levels. Assuming all levels have the same value for the learning-teaching rates, the academic program behavior can follow different patterns representing ideal learning and the extremes of weak and very effective learning.

Figure 7 illustrates these different patterns, where high relative values of $w_{i}$ represent very effective learning while low values yield weak learning.

It should be noted that the ideal learning corresponds to the achievement of final program outcomes of one hundred points or the full level scale. Also, if learning experiences are very effective (high value of $\mathrm{w}_{\mathrm{i}}$ ), students will achieve higher levels of learning outcomes over the one hundred points assumed for the ideal situation. If they are less effective or weak, the outcomes achieved are below the ideal situation.

Using a systems engineering point of view, this is typical expectation of open loop systems. In open loop systems the output performance will depend on the quality of the system components with no quality assurance (i.e. no feedback and adjustments) (Dorf, 1995) (Kilian, 2005).

To avoid this kind of open-loop situations in learning and teaching, and to comply with ABET or AACSB requirements, institutions introduce assessment, evaluations, and actions (i.e. feedback) for quality control and assurance.

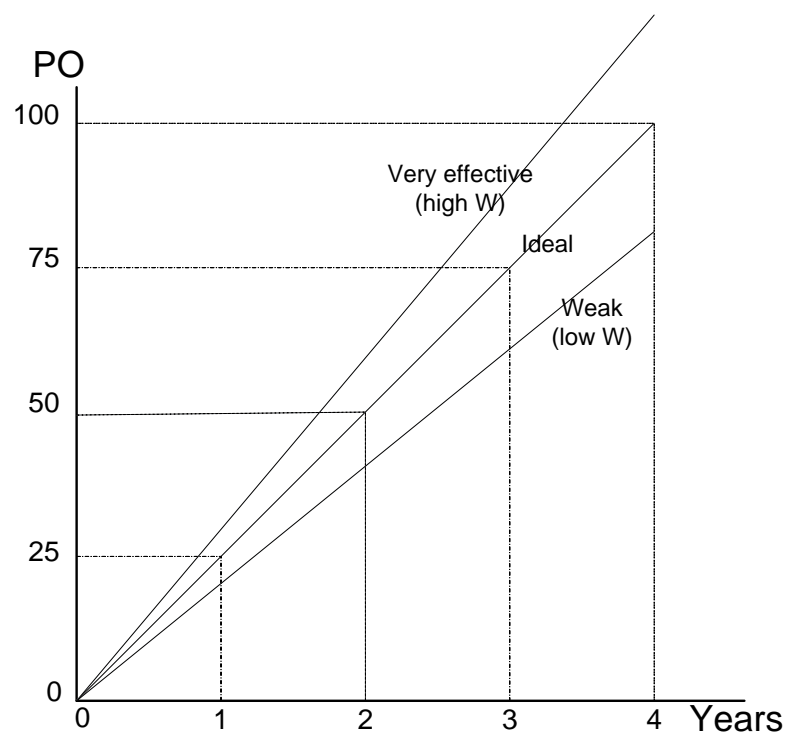

Figure 7: Effect of varying Teaching-Learning Effectiveness

The teaching and learning rate weight $w_{i}$ is a bounded number. It cannot be zero or negative i.e. $w_{i}>0$, due to the institutional policies and standards regarding faculty members' qualification which ensures some learning. There is also an upper ideal level for this $w_{i}$ weight which is not a limit, as institutions can have very effective learning with higher $w_{i}$ values. This ideal level corresponds to the achievement of twenty five points on the learning outcomes index scale for one academic program year or level. Although the collective academic year can be assumed to be made of three semesters of about 45 weeks in total, however, to simplify the analysis and later the 
dynamic simulation, we assume that students learning are spread over a whole year of 52 weeks. The estimate of ideal value of $w_{i}$ corresponds to the ideal learning situation. In this situation, the learning outcomes index achieves maximum value of twenty five points at the end of the year. Considering the integrator model assumed earlier, the ideal value for $w_{i}$ can be estimated to be close to 0.5 points /week.

Therefore, $0.5 \geq w_{i}>0$

In practice, institutions usually assess the learning outcomes of graduates $\left(P O=P O_{4}\right)$ and perform an evaluation of the data to determine effectiveness for self-improvement. It is a common practice for institutions to assume specific threshold or benchmark value (say 60\%) to determine whether a certain learning outcome is achieved. If an outcome is achieved, no actions are needed. However, if an outcome is below the benchmark value, an investigation is pursued in all relevant courses (especially fourth year courses), and actions will follow. Actions will be introduced in the lower level courses but at different emphases and weights as they have a long term effect. All these actions change the learning effectiveness in the considered courses, i.e., in this model it would change the value of $w_{i}$. The academic program for one level can be assumed to be a teaching and learning process which is an accumulation process. The input rate to this process is the teaching-learning rate. It depends on the teaching-learning effectiveness factors. The output from this process would add to the prior knowledge to form the students total learning which is in the model corresponds to the program learning outcomes index. This total learning, in turn, can enhance the teaching-learning rate through a positive reinforcing feedback loop as it contribute to the enhancement of information preparation among other factors (Eftekhar \& Strong, 2007). Thus, the teaching-learning rate can be changed through the actions of outcomes assessment feedback.

The total learning is represented as;

Total Learning $=$ Prior Knowledge + TLPO

Where, TLPO represents the Teaching-Learning Process Outcome. It can be expressed as;

$\mathrm{TLPO}=\int_{0}^{1 \text { year }}($ Teaching \& Learning Rate - Completion Rate $) \mathrm{dt}$

The teaching and learning rate represents the enrolling students, who enjoyed teaching and learning effectiveness. This depends on many education factors affected by the assessment feedback. The completion rate represents the graduating students from this academic level with learning achieved at the end of the year. The lower limit of the integration is zero, which represents the start of the academic year. The upper limit is one year which represents the end of the academic year. This implicitly assumes that the collective behavior and impact of certain academic year on the development of total learning is spread over the full year. This assumption will simplify the numerical simulation developed later. On the other hand, the assumption has some merits if it is assumed that the full academic experience of the students throughout the year contribute to the students total learning. The Teaching \& Learning Rate $w_{i}$ can be expressed as,

$\mathrm{w}_{\mathrm{i}}=$ Teaching Learning Effectiveness $+\mathrm{k} * \mathrm{PO}_{\mathrm{i}}$

The term $k * P O_{i}$ accounts for the positive feedback effect discussed earlier, where $\mathrm{k}$ is a gain factor to represent the relative effect of $P O_{i}$ on the learning process dynamics. The teaching learning effectiveness term is described as;

Teaching Learning Effectiveness = Initial Effectiveness + Feedback Actions

Where, the "Initial Effectiveness" term represents the teaching and learning effectiveness without any assessment feedback intervention. 


\section{The Dynamic Model of Assessment, Evaluation and Feedback Actions Model}

The assessments process is concerned with measuring the program learning outcomes at the end of the academic year or at a fixed point in time during the academic year. The results are analyzed and evaluated based on established criteria, and benchmark levels. Then, the faculty assessment committee plans feedback decisions or actions. These actions are communicated to the faculty teaching relevant courses, for implementation of these decisions or actions. These communications are carried out frequently during the academic year. This model development assumes that these assessments and communications are carried out only once at the end of the academic year for the integration model above. This is basically a feedback loop which is active only for a very brief period of time at the end of each academic year. For the rest of the year, the academic program is essentially an open loop system with no outcomes assessment feedback. The developed model and simulation captures this unique feature of the outcomes assessment feedback. The program outcomes index and its assessment are represented by the value reached for the total learning, at the end of the academic year. It is assumed that the feedback actions can only increase to the teaching and learning effectiveness. Hence, it increases the teaching and learning rate in the process. Feedback actions are not allowed to reduce the teaching and learning effectiveness as this would contradict the purpose of the academic institutions as well as ABET and AACSB requirements.

\section{The Complete Model}

These institutional assessments, evaluations, actions, policies, and mechanisms can be modeled as shown in the following Stock \& Flow diagram (Figure 8). In this diagram, a completion rate is added to indicate that at the end of the year the students will graduate from this academic level and the Stock (Accumulator) will be emptied (i.e. re-initialized to zero). It is worth noting that this complete model represents one academic level, group of courses, or even one course as discussed earlier.

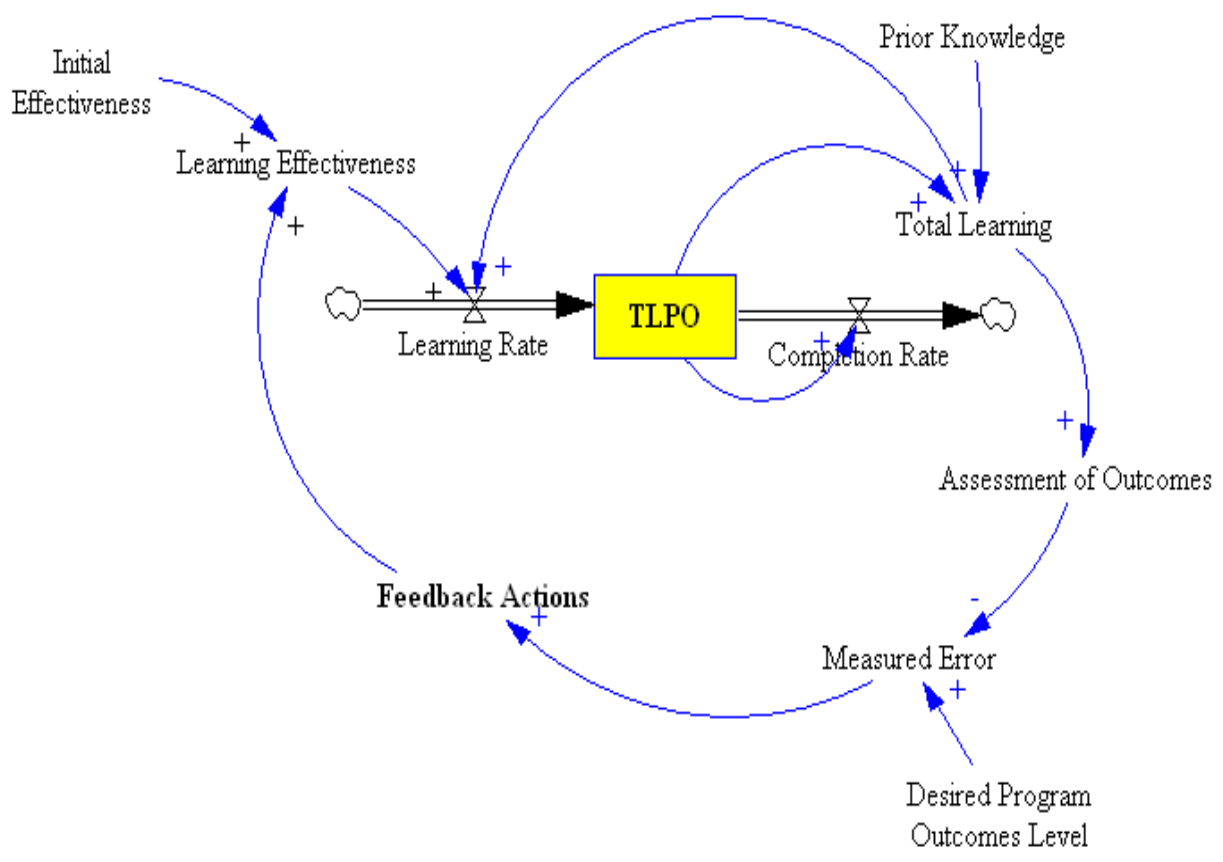

Figure 8: Stock \& Flow diagram of the complete model

\section{THE COMPUTER SIMULATIONS}

This section presents the quantitative part of the study namely the computer numerical simulation. This simulation will estimate the expected dynamic behavior in the long term. The model is built and simulated in a special system dynamics simulation environment. The full system equations are detailed in the Appendix. 


\section{VENSIM PLE Simulation Environment}

The completed model is simulated in VENSIM PLE (VENSIM, 2011) system dynamics simulations environment. VENSIM PLE is available for free download and use for academic and research purposes. The complete Stock \& Flow diagram is produced in VENSIM PLE. All developed equations are programed in this simulation software. The software has the ability to produce a report on all programed model equations. This report appears in the Appendix. A number of numeric variables limits and values have to be initialized. For example, the assumed full level for the outcomes index is set to 25 point as it represents one academic level out of four levels in the full program model. Moreover, it is assumed that the desired program outcomes level to achieve is 15 points which is $60 \%$ of the 25 points. This $60 \%$ threshold is the benchmark used at the College of Information Technology in University of Dubai (University of Dubai, 2011). This is also the benchmark approved by AACSB and ABET and used in many institutions worldwide. Alternatively, a higher benchmark level can be assumed and simulated if needed. Furthermore, the positive reinforcing loop is assumed to have a gain (i.e. power) of $1 \%$ of an ideal learning effectiveness. This means that its effect is modest and allows observing the dynamic behavior of the TLPO (Teaching Learning Process Outcome). A weak teaching learning situation is assumed in order to clearly examine the impact of the assessments feedback. A variety of assessment feedback actions levels are used to investigate its impact on the teaching-learning process in the long run.

\section{The Simulation Results}

Many simulation runs have been performed. However, we selected the key simulation runs which capture the discovered phenomena and reported them in Figure 9, Figure 10, Figure 11, Figure 12 and Figure 13. However, the full simulation model equations are reported in the Appendix to enable any interested researcher to re-build the simulation and experiment with many simulation runs. The simulation results presented in Figure 9 confirm the growth nature of the total learning from an initial level, which is the prior knowledge level, to the final level at the end of the academic year.

\section{Total Learning}

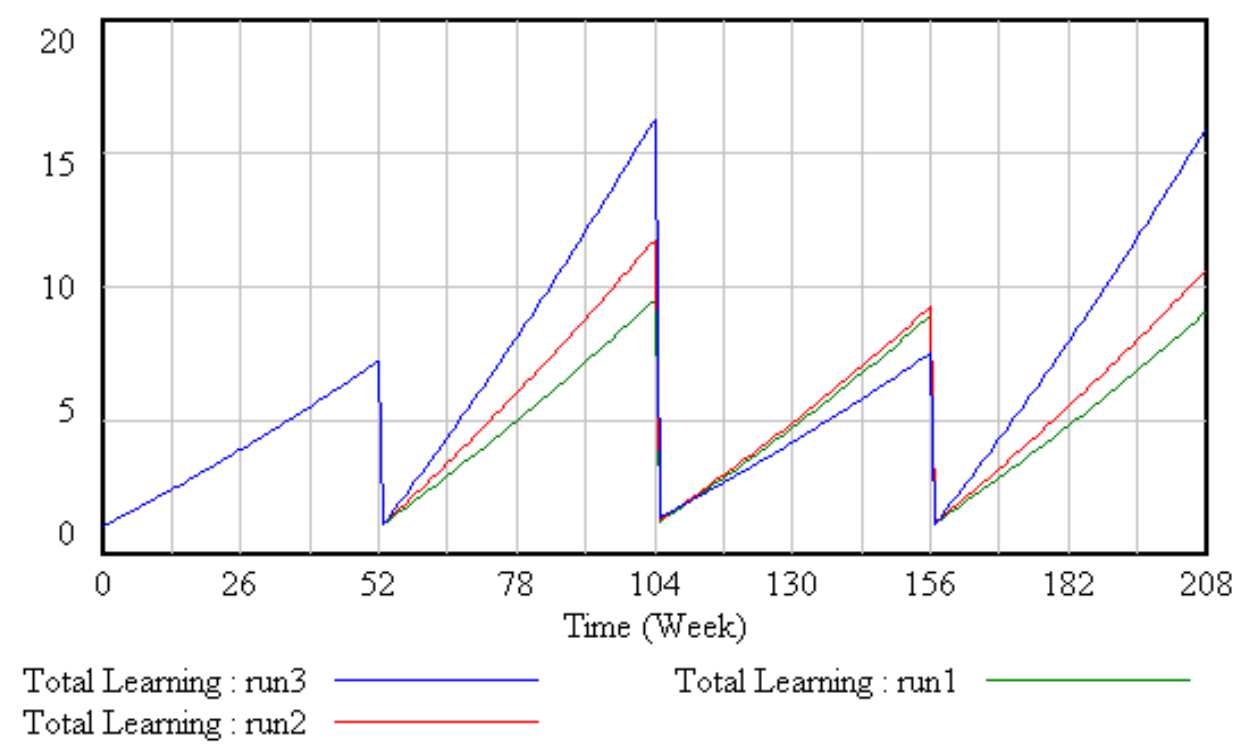

Figure 9: VENSIM PLE Simulation of Dynamic behavior of Total Learning in the same academic level

For the first academic year, there is no assessment feedback. The result for all simulation runs coincide with each other. The final learning level reached is 7 points (i.e. very modest) which is well below the benchmark 
(i.e. desired level) of 15 points. At the end of the year this learning is assessed and used in the feedback. Different feedback levels are assumed which would alter the teaching and learning rate in different levels. These feedback actions are calculated as proportional to the measured outcomes error (i.e. proportional decisions). In the first simulation run with the first level of feedback the learning reached by the end of the $2^{\text {nd }}$ year (i.e. $2^{\text {nd }}$ delivery of the same academic level courses) is almost 9 points (i.e. modest improvement). However, in the second simulation run with higher feedback actions, the level reached is almost 11 points. In the third simulation run with the highest level of feedback actions considered, the learning reached almost 16 points and satisfies the desired benchmark level. This dynamic simulation results confirm the importance and value of implementing assessment feedback to improve the quality of the academic programs. However, at the end of $3^{\text {rd }}$ year, the total learning drops for all considered levels of assessment feedback actions. Remarkably, it is followed by significant increases at the end of $4^{\text {th }}$ year. These results can be summarized by extracting the end of year Program Outcomes values and plotting them versus the years as shown in Figure 10 and Figure 11. While Figure 10 display the results achieved without assessments feedback, Figure 11 shows the dynamic behavior with assessments feedback closing the loop on the academic program level.

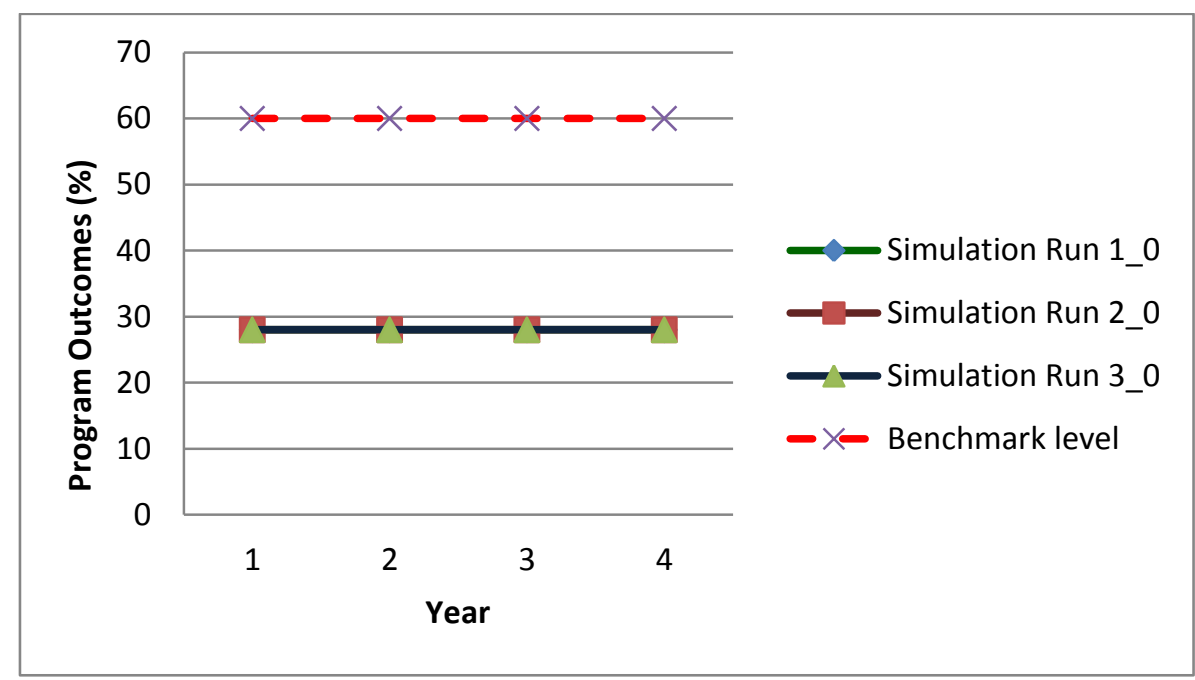

Figure 10: Program Outcomes Achieved Without Assessments Feedback

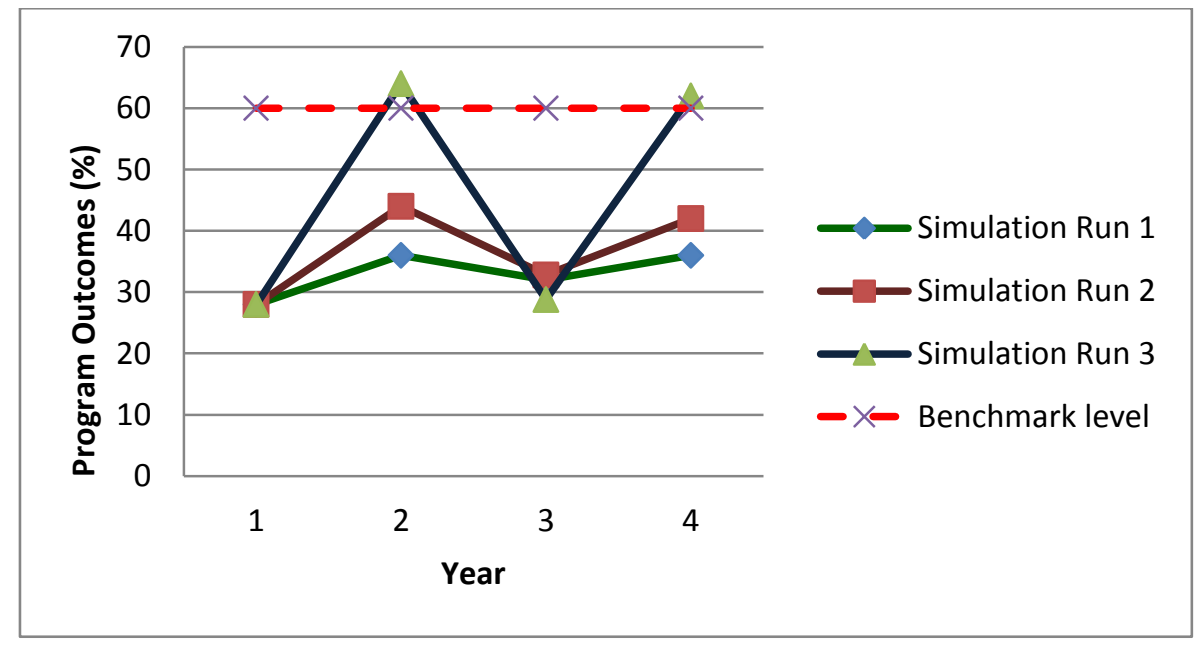

Figure 11: Program Outcomes Achieved with Assessments Feedback 
These results (as shown Figure 11) reveal that the program outcomes levels achieved at the end of the academic year with assessment feedback are not stable at a certain level. In fact, they exhibit significant sustained oscillations. The simulation runs corresponding to strong assessment feedback have the program outcomes oscillating around the benchmark level $(60 \%)$. However, the simulation runs corresponding to weak assessment feedback have the program outcomes oscillating well below the benchmark level. We name this discovered oscillation as Learning-Assessment Oscillation.

\section{DISCUSSIONS}

Generally speaking, this discovered Learning-Assessment Oscillation is created through the introduction of the assessment feedback loop as program outcomes without assessments feedback are stable and not oscillating as evident in practice and demonstrated in Figure 10. In addition, this can be attributed to the model developed above where there is no consideration for a memory part. This memory part should retain changes made in the course teaching learning effectiveness based on the feedback actions from one academic year to another. This unintentional modeling limitation illustrated the importance and need for such provision, which may be termed Course Memory. Other implemented simulation runs performed with Course Memory implemented to retain all assessments changes show that these learning oscillations can be greatly reduced. Furthermore, experimenting simulation runs with a memory part integrated in the assessment feedback (i.e. Assessment Memory) were performed. The assessment memory makes the feedback actions depend, beside the current assessment results, on the assessment results of the past two or three years but with less weighting. Figure 12 present the simulation results with assessment memory implemented as integration of equal weight to all past three years. It has the profound impact of eliminating the learning-assessment oscillation as observed in Figure 11.

\section{Total Learning}

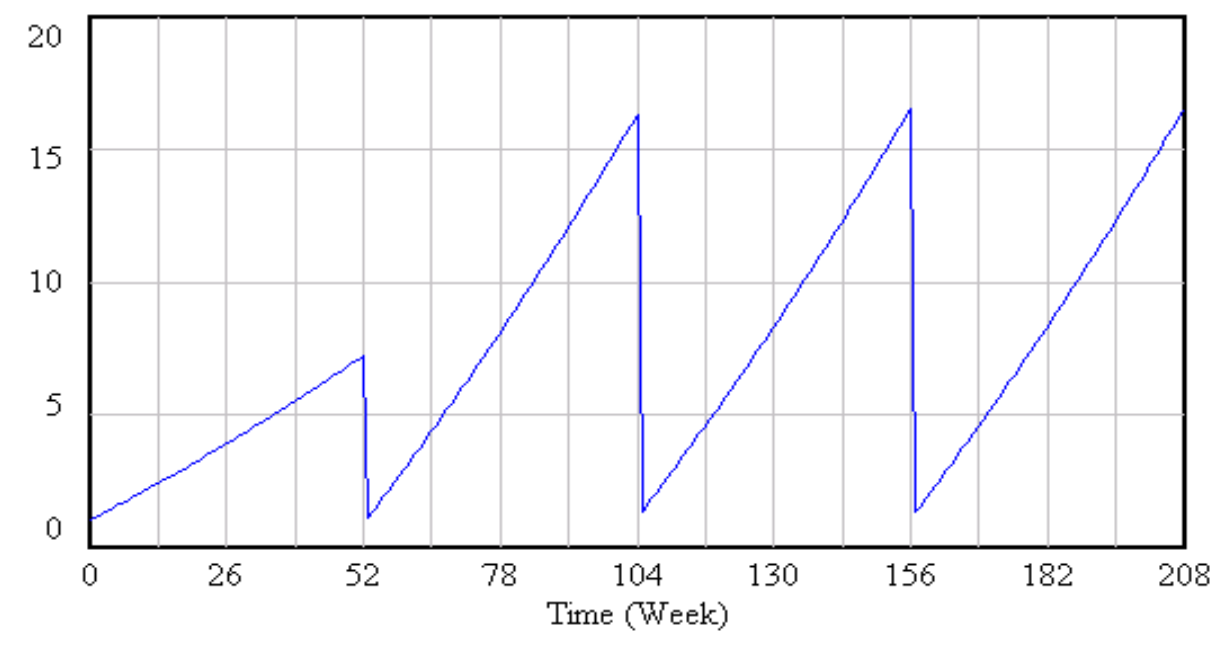

Total Learning : run21

Figure 12: VENSIM PLE Simulation of Dynamic behavior with Assessment Memory

These program outcomes results at the end of the years can be extracted from the results shown in Figure 12 and represented as shown in Figure 13 below to show only the program outcomes level at the end of the academic year/level. 


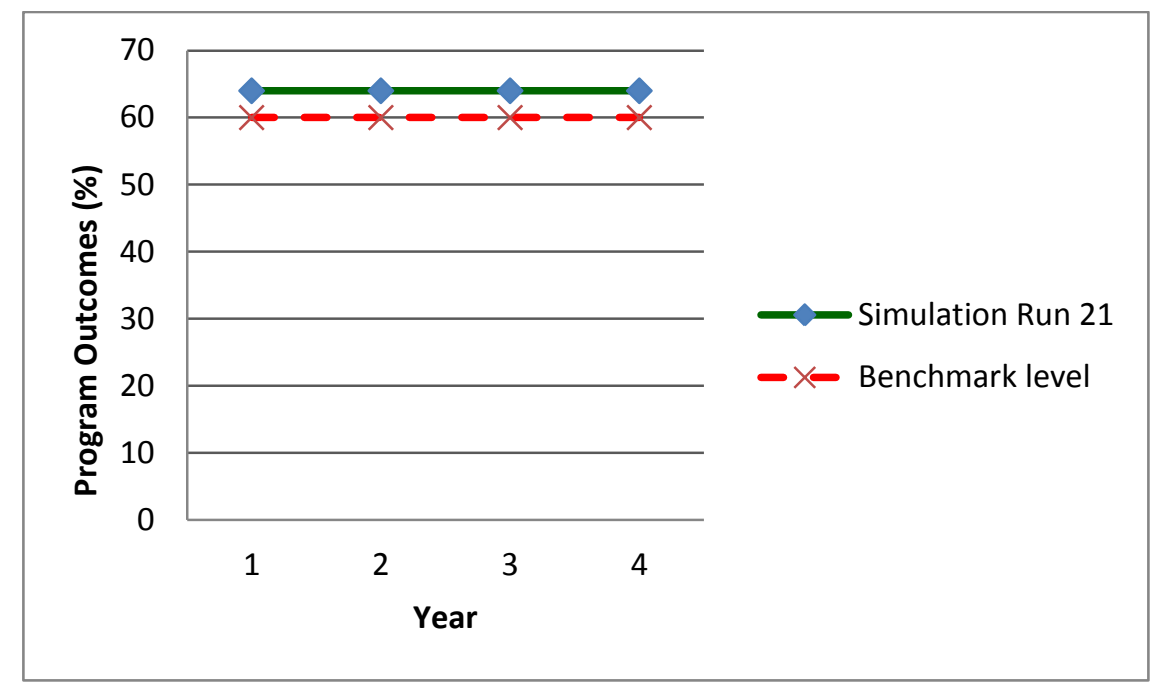

Figure 13: Program Outcomes Achieved with Assessments Memory in the Feedback Loop

Furthermore, in all simulation runs, the considered time horizon is limited to four academic years. The reason is that many academic institutions tend to redesign their programs' curricula every three to five years to keep their programs up-to-date and attractive. In these simulations, the placed upper limit on the feedback actions is close to the level of the ideal teaching and learning effectiveness value. This is imposed, as it is not logical to expect assessment feedback actions to create teaching and learning environments, procedures, delivery, etc. beyond an ideal situation.

\section{VALIDATION}

To validate our developed model, results and discovered oscillation, we analyze and study the actual assessment data collected over five year period at the College of Information Technology (CIT), BSc in Computing and Information Systems (CIS) (CIT Assessments Committee, 2010) (CIT Assessments Committee, 2011). The assessment instrument used is a benchmark exit exam developed collectively by the CIT faculty to assess the CIS final program outcomes among other instruments used for the same purpose. Generally speaking, this assessment instrument is usually used in conjunction with an array of other instruments to produce final estimates of the program outcomes. However, this particular instrument is chosen and used in this analysis as it will help in validating the study.

The exit exam is administered to the graduating students during the 400 level course of Capstone Project. The exit exam is composed of 80 multiple choice questions which assess eight knowledge areas and their corresponding eight program outcomes. The questions are extracted from a limited number of courses which were deemed crucial for the achievements of the program outcomes. Therefore, the results of this exam can be regarded as assessing the outcomes from an academic unit representing the collective behavior of a chosen number of courses in CIS curriculum. It is important to study this collective behavior and compare it with that obtained earlier in the simulation of one academic unit behavior.

Each program outcome is assessed by counting the number of students who achieved it and normalizing the result as a percentage number. If the obtained percentage is higher than $60 \%$, the outcome is declared as achieved and no feedback actions are planned or considered. However, if the outcome is below $60 \%$, the assessment committee conducts a careful investigation and consequently recommends specific actions to be considered. These actions are usually communicated to the faculty member teaching the specific courses which are deemed critical for the achievements of these outcomes. The responsibility of the implementation is mainly the concerned faculty responsibility. In many situations, a new faculty member inherits the course and finds that he is responsible for implementing these changes. Although faculty members usually share a good part of their course files, it is found 
that new faculty teaching will always bring significant changes in their course delivery compared with the initial faculty who taught the course in the previous year. Sometimes there is a breakdown in communication to the new faculty about the assessments committee recommendations. In other situation, they might just forget about these recommendations. Sometimes, the situation is more drastic as some 100 and 200 level courses are taught by parttimes who usually have a limited attention and focus on students and curriculum needs. The responsibility of proper communication and implementation follow up is with the Dean of the College. This task proved to be daunting and the Dean tends to be occupied with more urgent or strategic issues such as planning new programs.

To further study the collected data, a program outcomes index is calculated by averaging the percentage values of all eight outcomes for the specific year. This approach is reasonable as the exam does not seem to weigh an outcome more than the other and all outcomes at the end are normalized and presented as percentage values. It must be noted that same outcomes scored above the benchmark level and are acceptable. While others scored below the benchmark level. The program outcomes index would average these results.

Table 1 shows the results obtained of the program outcomes index for five year period from the academic year 2006-2007 till the academic year 2010-2011 (CIT Assessments Committee, 2010) (CIT Assessments Committee, 2011).

Table 1: CIS Program Outcomes Index for five year period based on Exit Exam

\begin{tabular}{|l|c|c|c|c|c|}
\hline Year & $\mathbf{1}$ & $\mathbf{2}$ & $\mathbf{3}$ & $\mathbf{4}$ & $\mathbf{5}$ \\
\hline Program Outcomes Index (\%) & 46.0 & 47.8 & 43.5 & 42.8 & 45.7 \\
\hline
\end{tabular}

Figure 14 displays a graphical representation of these data to be able to visualize its trend. It is quite evident that the results are not stable at a fixed level and they exhibit an oscillation. This is phenomenon can be explained by referring to the assessment scenario in CIT which have been explained above. This scenario clearly demonstrates modest implementation of the assessments feedback actions.

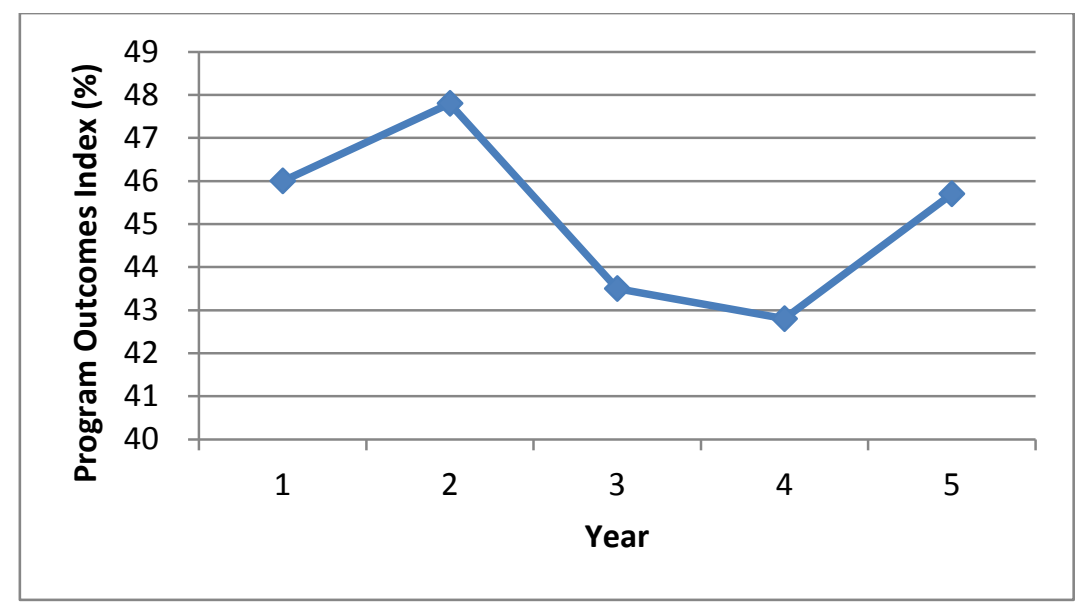

Figure 14: The CIS Program Outcomes Index measured over five year period based on Exit Exam

Furthermore, the changes in the faculty teaching assignments and use of part-timers confirm a modest existence of the term we called before a Course Memory. We can deduce that the modest implementation of assessments feedback actions is largely responsible for the obvious drop below the benchmark level of $60 \%$. While, the modest course memory is largely responsible for the oscillatory behavior. The oscillatory behavior observed in these results seems to have a longer period than the simulations results with assessments feedback appearing in Figure 11. This can be explained as the simulations represented result of somehow ideal system. However, the actual 
data obtained represent the reality of a complex system where many courses are involved at varying levels of assessments feedback and having some forms of course memories spread through these courses. These salient differences definitely would have the effect of attenuating the oscillatory behavior both in amplitude and period i.e. it would spread over slightly more years than expected in simulation.

These results represent strong indicator to the validity of the developed system dynamics model and the simulation results presented in the previous section. These results can be a good motivator to pursue development of more complicated models and further comparison with actual assessments data.

\section{CONCLUSIONS}

This study provides a one level system dynamics model of an academic program with outcomes assessment based feedback. Academic programs are complex social systems. Adding assessment feedback further increases this complexity and adds new modes of dynamic behavior. This study focuses on the dynamic behavior of one academic level with assessment feedback. This is regarded as the fundamental building block for the full dynamic model as discussed and illustrated with two main general structures for assessment feedback implementation; a centralized assessment and a distributed assessment. The developed model is basically two inputs - one output system. The inputs represent the students' prior knowledge and background, and the teaching and learning effectiveness factors. The single output can represent a single outcome or represent a unified program outcomes index. The model is built and tested in a system dynamics simulation environment which can help academic administrators study the consequences of any assessment policy decisions in an interactive and dynamic manner. It can be used to study the effects of different assessment feedback policies such as proportional actions versus integration actions using past assessment.

The simulation of the dynamic behavior demonstrates the existence of a Learning-Assessment Oscillation. This oscillatory behavior is not an intrinsic feature of academic programs in general. However, it is created as the assessment feedback closes the loop on the academic program system which is basically an accumulation causing time delay. The feedback actions corresponding to this oscillation are proportional actions (i.e. proportional to the measured error). This is another reason for the oscillatory behavior. As the feedback actions are strong when measured errors are large, they create strong correction and cause outcomes to reach higher level at the end of the academic year. This in turn causes a much smaller error which calls for a much weaker feedback actions and adjustments in the following year. Hence, the outcomes achieved in the following delivery year will drop significantly. This oscillation can only be mitigated if procedures and policies are implemented to retain changes and improvements in course teaching and learning effectiveness such as course delivery instructions, (Course Memory) and / or implementation of feedback actions which make use of past years (two or three years) assessment results (Assessment Memory). This indicates the institutional need to establish policies and practices to create and maintain Course Memory activities. Institutional polices should be developed to mandate proper training of faculty members for best assessments practices as well as use of course files, possibly through Learning Management Systems (LMS) and the emerging cloud technology in order to ensure implementation and retention of all recommended assessments feedback actions. Or alternatively adopt innovative teaching methods enabled through clever adoption of technology to enhance the students learning experiences and retain all faculty changes and additions to the course similar to the approach developed in (Mackie \& Liu, 2010). On the other hand, academic administrators can develop a policy of using peer class visit to help in this regard. Otherwise, they can request the Dean to conduct midterm meetings with the faculty as well as a visit to the faculty classes for assurance of implementation of all assessments recommendations. All these policies can result in an effective curriculum delivery and stable program outcomes as deemed necessary by ABET and AACSB standards.

A Memory part such as Course Memory can be perceived as a must in many System Dynamics models. However, its unintentional absence in the initial developed model allowed to clearly observing the LearningAssessment oscillation behavior. This in turn supports the value of course memory relation to good teaching and learning practice. This oscillation can appear in reality when a capable and experienced professor is replaced by a part-timer who is just a knowledge expert with weak teaching experience to teach specific course as part of cost cutting measures and policies observed in many academic institutions. In addition, policies and practices to implement Assessment Memory (i.e. use of past and recent assessment recommendations as an integration-based 
assessment feedback policy) are recommended to be developed and integrated in assessment feedback as part of the institutional effectiveness framework. The presented results which simulated feedback actions based on integration of current as well as past years actions, show great improvements in outcomes with elimination of the oscillatory behavior. Nonetheless, the assessment structures and simulation results indicate the need to embed outcomes assessment in all curriculum courses or for every academic year or group of courses based on a model academic plan. This would require designing program outcomes specific level for each academic level, and performing outcomes assessment at every academic level. Hence, evaluation and actions (i.e. feedback) are taken at every academic year level. The simulation results support these recommendations. This is a novel approach to study assessments processes using system dynamics approach. Furthermore, although this study originated from experiences with the successful accreditation of a Computing \& Information Systems program and further validated by its data, it can be easily generalized to other specializations and levels of academic programs.

\section{RECOMMENDATIONS FOR FUTURE RESEARCH}

This study can be used to develop a full academic program model for any outcome assessment feedback structure. The full dynamic model of any four year academic program is a highly complex social system with high number of variables and parameters. Its development requires considerable resources. This study is the first step and corner stone toward this ultimate goal, which would provide academic administrators the power to design and test assessment polices for higher academic achievement. A natural follow up study could look into detailed investigation of best practices which implements a strong course memory such as recommendations on the course file and its use in the course delivery, stabilizing teaching assignments for faculty members to enhance the course memory and all the recommended policies delineated in the previous section. Another ambitious study can attempt building the full model using the model and results presented in this work and calibrate the model for specific institutional settings to enable testing academic policies for this particular academic institution.

\section{AUTHOR INFORMATION}

Hussein Fakhry is the Assistant Dean of the College of Information Technology at the University of Dubai. He received $\mathrm{PhD}$ in Intelligent Control Systems and Robotics from the University of Waterloo, Canada. His research interests are in Information Systems Research using System Dynamics, Information Systems Security, E-Commerce and E-Business, Decision Support Systems, Applications of Artificial Intelligence, and Assessment of Academic Programs. His research appeared in international journals and conferences such as Review of Business Information Systems, Communications of the International Information Management Association (CIIMA), An International Journal of Information \& Security, The Journal of Mathematical and Computer Modeling, Proceedings of the IEEE International Conference on Information and Communication Technologies, and Proceedings of the IADIS International Conference on Information Systems. E-mail: hfakhry@ud.ac.ae

\section{REFERENCES}

1. AACSB. (2011). The Association to Advance Collegiate Schools of Business, Accreditation Standards. Retrieved 19, 2011, from AACSB: http://www.aacsb.edu/accreditation/standards/

2. AALHE. (2011). Asociation for the Assessment of Learning in Higher Education. Retrieved 1 5, 2011, from http://aalhe.org/

3. ABET. (2011). The Accreditation Board For Engineering and Technology, Criteria for Accrediting Computing Programs, 2012 - 2013. Retrieved 1 7, 2011, from ABET: http://www.abet.org/computingcriteria-2012-2013/

4. Allan, J. (1996). Learning Outcomes in Higher Education. In J. Allan, Studies in Higher Education, Vol. 21 (pp. 93 - 98).

5. Allen, M. J. (2004). Assessing Academic Programs in Higher Education. Bolton, MA: Anker.

6. Angelo, T. (1995). Assessment. Bulletin. American Association of Higher Education.

7. CDU. (2009). Academic Program Review Handbook for: 1. Annual Program Assessment (learning outcomes) 2. Annual Self-Review 3. External Peer-Review. Charles Drew University Of Medicine And Science. 
8. CIT Assessments Committee. (2010). CIT Annual Assessment Report 2009-2010. Dubai: College of Information Technology, University of Dubai.

9. CIT Assessments Committee. (2011). Capstone Project Course Assessments Report. Dubai: College of Information Technology, University of Dubai.

10. Dorf, R. C. (1995). Modern Control Systems. Boston, MA: Addison-Wesley.

11. Eftekhar, N., \& Strong, D. (1998). Towards Dynamic Modeling of a Teaching/Learning System Part 2: A New Theory on Types of Learning. International Journal of Engineering Education, Vol. 12, No. 6., pp. 388-406.

12. Eftekhar, N., \& Strong, D. R. (1999). Towards Dynamic Modeling of a Teaching/Learning Systems Part 3: The Simulation Model. International Journal of Engineering Education, Vol. 15, No. 3, pp. 168 - 190.

13. Eftekhar, N., \& Strong, D. R. (2007). Dynamic Modeling of a Learning Process. International Journal of Engineering Education.

14. Eftekhar, N., Strong, D. R., \& Hawaleshka, O. (1997). Towards Dynamic Modeling of a Teaching/Learning Process: 1- The Unified Theory. International Journal of Engineering Education, Vol. 12, No. 6.

15. Fincher, C. (1994). Learning Theory and Research. In K. A. Feldman, \& M. B. Paulsen, Teaching and Learning in the College Classroom. ASHE Reader Series, Ginn Press, MA.

16. Forrester, J. W. (1958). Industrial Dynamics: a major breakthrough for decision makers. Harvard Business Review, No. 36, pp. 37 - 66.

17. Forrester, J. W. (1990). The beginning of system dynamics. Boston, MA: International System Dynamics Society.

18. Gloria, R. (2006, December). Assessment Tips. Community Matters. A Monthly news letter of the ABET Community.

19. Kilian, C. (2005). Modern Control Technology. Thompson Delmar Learning.

20. Lane, D. C. (2007). The Power of the Bond Between Cause and Effect: Jay Wright Forrester. System Dynamics Review, 23 (2-3): 95 - 118.

21. Mackie, B., \& Liu, C. (2010). Student-Centered, Student-Edited Online Course Materials: Teaching Approach for an Advanced Technical Course. Communications of the IIMA, Volume 10 Issue 3 pg. 59-67.

22. March, P. A. (2007). What is known about Students Learning Outcomes and How does it relate to the Scholarship of Teaching and Learning. International Journal for the Scholarship of Teaching and Learning, 23 - 34.

23. Morante, E. A. (2003). A Handbook on Outcomes Assessment for two year Colleges. College of the Desert, California Community College Chancellor's Office.

24. UCF. (2008). Program Assessment Handbook. University of Central Florida.

25. University of Dubai. (2011). Process Manual. Dubai: University of Dubai.

26. VENSIM. (2011). User's Guide. Retrieved 1 5, 2011, from VENSIM: http://www.vensim.com/documentation.html

27. Walvoord, B. A. (2004). Assessment clear and simple: A practical guide for institutions, departments and general education. San Francisco: Jossey-Bass. 


\section{APPENDIX: VENSIM PLE SIMULATION EQUATIONS WITH ASSESSMENT MEMORY}

(01) Assessment of Outcomes $=($ Total Learning $* \operatorname{PULSE}(52,1))+($ Total Learning $* \operatorname{PULSE}(104,1))+($ Total Learning * PULSE $(156,1))$ Units: Dmnl

(02) Completion Rate $=(\operatorname{TLPO} * \operatorname{PULSE}(52,1))+(\operatorname{TLPO} * \operatorname{PULSE}(104,1))+(\operatorname{TLPO} * \operatorname{PULSE}(156,1))$ Units: Dmnl

(03) Desired Program Outcomes Level=15* (PULSE(52,1) + PULSE(104,1) + PULSE(156,1)) Units: Dmnl

(04) Feedback Actions $=$ INTEG $(0.02 *($ MAX $($ Measured Error, 0$)), 0)$ Units: Dmnl $[0,0.48]$

(05) FINAL TIME $=208$; Units: Week

(06) Initial Effectiveness=0.1; Units: Dmnl

(07) INITIAL TIME $=0$; Units: Week

(08) Learning Effectiveness=Initial Effectiveness + Feedback Actions; Units: Dmnl

(09) Learning Rate $=0.005 *$ Total Learning + Learning Effectiveness; Units: Dmnl

(10) Measured Error=Desired Program Outcomes Level - Assessment of Outcomes; Units: Dmnl

(11) Prior Knowledge=1; Units: Dmnl

(12) SAVEPER = TIME STEP; Units: Week

(13) TIME STEP $=1$; Units: Week

(14) TLPO= INTEG (Learning Rate - Completion Rate, 0); Units: Dmnl [0,30]

(15) Total Learning=TLPO + Prior Knowledge: Units: Dmnl 
NOTES 